Hautarzt 2020 · 71:4-5

https://doi.org/10.1007/s00105-019-04524-y

(c) Springer Medizin Verlag GmbH, ein Teil von Springer Nature 2019

\section{Michael Jünger}

Dermatologie und Venerologie, Universitätsmedizin Greifswald, Greifswald, Deutschland
Erkrankungen des venösen und lymphatischen Kreislaufs führen Patient ${ }^{\star}$ Innen in betriebswirtschaftlich relevanter Häufigkeit in dermatologische und zunehmend chirurgische Praxen. Innovationen ermöglichen eine effektive und effiziente Therapie, erleichtern den Betroffenen die Therapiephase mit nachhaltiger Linderung der Beschwerden. Daher ist die Zusatzbezeichnung Phlebologie nicht nur medizinisch von Nutzen. Dank des Ärztetages 2019 ist der Zugang zu dieser Zusatzbezeichnung nun auch für die KollegInnen möglich, die bereits in eigener Praxis tätig sind und nicht bei einem Weiterbildungsbefugten Kenntnisse und Erfahrungen erwerben können. In 5 Beiträgen dieser Ausgabe wird der aktuelle Stand chirurgischen, interventionellen und konservativen Vorgehens kompakt auf den klinischen Alltag ausgerichtet dargestellt.

\section{》) Der aktuelle Stand chirurgischen, interventionellen und konservativen Vorgehens wird kompakt dargestellt}

Die dank Duplexsonographie auf die venöse hämodynamische Störung gezielt ausgerichtete Phlebochirurgie in Form von Krossektomie und Exhairese insuffizienter intra- und extrafaszialer Venensegmente beseitigt bei zutreffender Indikationsstellung sicher stauungsbedingte Beschwerden, trophische Hautveränderungen inklusive Ulcus cruris venosum heilen $a b$, ohne unerwünschte Folgen auszulösen. Neben den klassischen chirurgischen Verfahren haben sich die katheterbasierten thermoablativen Methoden etabliert. Da diese mit lokalen Anästhesieverfahren durchführbar sind, bieten sie sich insbesondere für Patient ${ }^{\star}$ Innen mit Komorbiditäten an. Sie lassen sich auch unter den Bedingungen einer gerinnungshemmenden Therapie durchführen. Die hämodynamische Verbesserung des klassischen chirurgischen Vorgehens und der katheterbasierten Verfahren unterscheiden sich nicht. Erneute venöse Refluxe im späteren Verlauf sind bei den oft älteren Patient ${ }^{\star}$ Innen ohne klinische Relevanz.

In der Hand geübter Sklerotherapeut ${ }^{\star}$ Innen werden bei Vorliegen großlumiger Varizen zumindest initial hämodynamische Verbesserungen erzielt, die den chirurgischen Verfahren nicht nachstehen. Dieses kosteneffiziente Verfahren eignet sich für Patient ${ }^{\star}$ Innen, die einer zeitlich längeren Therapiephase mit wiederholten Interventionen offen gegenüberstehen. Die sklerosierende Wirksamkeit wird mittels Aufschäumen des Verödungsmittels Polidocanol erhöht, sodass wesentlich längere Venenabschnitte in einer Therapiesitzung erreicht werden können, ausschließlich unter Ultraschallkontrolle zur Vermeidung von Komplikationen. Mit der Schaumsklerosierung lassen sich zudem angiodysplastische Malformationen und Lymphfisteln ausschalten.

Unmittelbar nach operativer oder interventioneller Ausschaltung der venösen Refluxe reduziert die Kompressionstherapie Schmerzen, verhindert Komplikationen und trägt zur Nachhaltigkeit der Maßnahme bei. Wird diese exzentrisch durch Aufpolsterung im Verlauf der behandelten Venensegmente ausgeführt, erhöht sich die Wirksamkeit. Steht die Kompressionstherapie nicht im Zusammenhang mit einer Intervention und ist auf Dauer ausgerichtet, steht die 
Anwendbarkeit für die Patient ${ }^{\star}$ Innen im Vordergrund. Die im deutschsprachigen Raum dominierende Empfehlung $\mathrm{zu}$ höheren Kompressionsklassen, v. a. der Klasse 2, sollte überdacht werden, ältere Menschen mit muskuloskeletalen Handicaps können medizinische Kompressionsstrümpfe der Klasse 1 anlegen und profitieren klinisch davon erheblich. Diese Strümpfe sind zulasten der gesetzlichen Krankenkassen rezeptierbar. Zahlreiche Vergleichsstudien zeigen mit hoher Evidenz den klinischen Nutzen von Zweikomponentenkompressionsstrümpfen für die Abheilung venöser Ulcera cruris auf. Orthesenartige Klettverschlussfertigverbände können an die Stelle der Zweikomponentenkompressionsstrümpfe treten. Die Betroffenen verwenden diese in Eigenregie, sodass keine Abhängigkeit von Pflegediensten besteht.

Im Therapiekonzept von Menschen mit fortgeschrittener chronischer venöser Insuffizienz und mit Lymphödem steht die Physiotherapie an prominenter Stelle. Manuelle und apparative Lymphdrainage mit intermittierend pneumatischer Kompression sowie die Mobilisierung der Gelenke der unteren Extremität, insbesondere des Sprunggelenkes, stehen dabei im Zentrum.

Ich wünsche Freude und praktischen Nutzen durch die Beiträge des Schwerpunktheftes Phlebologie/Lymphologie.

\section{Korrespondenzadresse}

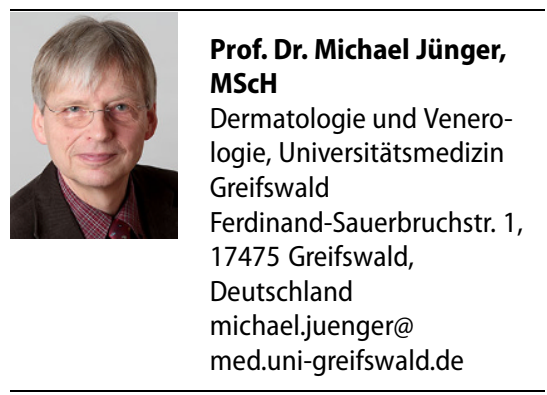

Interessenkonflikt. M. Jünger gibt an, dass kein Interessenkonflikt besteht.

\section{Möchten Sie einen Beitrag für die Zeitschrift Der Hautarzt einreichen?}

Wir freuen uns, dass Sie unsere Zeitschrift Der Hautarzt mitgestalten möchten. Um Ihnen bei der Manuskripterstellung behilflich zu sein, haben wir für unsere Autoren Hinweise zusammen gestellt, die Sie im Internet finden unter www.DerHautarzt.de (Für Autoren).

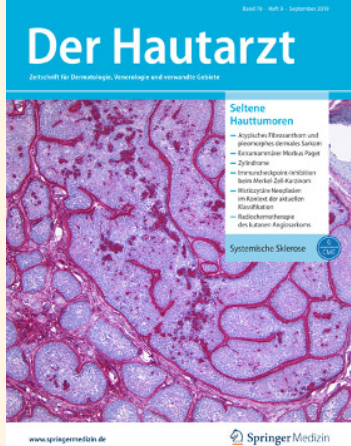

Bitte senden Sie Ihren fertigen Beitrag an:

\section{Originalien/Übersichten/}

Kasuistiken/Wie lautet Ihre Diagnose?

Prof. Dr. Daniela Bruch-Gerharz

Hautklinik des Universitätsklinikums

Düsseldorf, Moorenstr. 5,

40225 Düsseldorf

sdgehrke@googlemail.com

\section{CME Zertifizierte Fortbildung:}

Anfragen an:

\section{PD Dr. Evelyn Gaffal}

Universitätshautklinik

Universitätsklinikum Magdeburg

Labor für Experimentelle Dermatologie

Leipziger Straße 44

39120 Magdeburg

evelyn.gaffal@med.ovgu.de

Prof. Dr. Michael Meurer

Stiftung zur Förderung der

Hochschulmedizin

in Dresden

Fetscherstraße 74,

01307 Dresden

michael.meurer@uniklinikum-dresden.de
Prof. Dr. Sonja Ständer

Klinik und Poliklinik für Hautkrankheiten, Universitätsklinikum Münster

Von-Esmarch-Str. 58

48149 Münster

sonja.staender@uni-muenster.de

Prof. Dr. Rolf-Markus Szeimies

Klinik für Dermatologie und Allergologie, Knappschaftskrankenhaus Recklinghausen, Klinikum Vest $\mathrm{GmbH}$,

Dorstener Str. 151,

45657 Recklinghausen

dermatologie@kk-recklinghausen.de

PD Dr. Dr. Alexander Zink

Klinik und Poliklinik für Dermatologie und Allergologie am Biederstein,

Technische Universität München

Biedersteiner Str. 29

80802 München

alexander.zink@tum.de

\section{In der Diskussion:}

Prof. Dr. Alexander Kapp/

Prof. Dr. Thomas Werfel

Klinik für Dermatologie, Allergologie

und Venerologie,

$\mathrm{MHH}, \mathrm{OE} 6600$,

Carl-Neuberg-Str. 1, 30625 Hannover

derma@mh-hannover.de

\section{Dermatoskopie - Fall des Monats:}

Prof. Dr. Andreas Blum

Hautarztpraxis Konstanz und Lehrpraxis der

Medizinischen Fakultät Universität Tübingen

Augustinerplatz 7

78462 Konstanz

a.blum@derma.de

\section{Rezepturtipp für die Praxis aus der Praxis:}

Bitte schreiben Sie bei Fragen an:

hautarzt@dac-nrf.de 